\title{
Das neue Flüchtlingsgesetz in Südafrika
}

\author{
Von Tamru Melese*
}

\section{Einleitung}

Von den sechziger bis Anfang der neunziger Jahre galt das südliche Afrika neben dem Horn von Afrika als einer der Brennpunkte der Flüchtlingsbewegungen in Afrika. Aufgrund der dramatischen politischen Veränderungen in der Welt in den letzten Jahren nach dem Ende des Ost-West-Konflikts gilt das südliche Afrika nicht mehr - abgesehen von den durch den andauernden Bürgerkrieg in Angola immer noch zur Flucht gezwungenen Menschen - als der Schwerpunkt der Fluchtbewegung. Man beobachtet seit einigen Jahren die Verlagerung der massiven Flüchtlingsbewegung vom nordöstlichen und südlichen Teil des Kontinents in die westlich und zentral gelegenen Regionen.

Die Hauptursache dafür, daß das südliche Afrika zu einem der Flüchtlingsherde wurde, war - neben den kolonialen Befreiungskämpfen gegen die Portugiesen in Angola, Mosambik und dem Befreiungskampf in Rhodesien in den siebziger Jahren - die Destabilisierungspolitik des Apartheidregimes ${ }^{1}$ vor der Transformation des politischen und gesellschaftlichen Systems Südafrikas. Die verstärkten Bemühungen der Apartheid, die Nachbarstaaten zu destabilisieren, geschahen in unterschiedlichen Formen: Man bediente sich dabei ökonomischen Drucks. Die Besetzung Namibias, die direkte und indirekte Gewaltanwendung gegen die Nachbarstaaten Südafrikas, insbesondere Angola ${ }^{2}$ und Mosambik ${ }^{3}$, und die Unterstützung oppositioneller Rebellen und Gruppierungen mit dem Ziel, Basen von ANC (African National Congress) und PAC (Pan African Congress) in den damals kommunisti-

* Diese Arbeit geht auf die freundliche Anregung meines Doktorvaters, Prof. Dr. Brun-Otto Bryde, zurück.

1 Richter, Roland, Flüchtlingsbewegungen und Zwangsmigration im südlichen Afrika. Ursachen und Dimensionen, 1991, S. 8.

2

Aufgrund der direkten und indirekten Unterstützung der UNITA durch das Apartheidregime haben Ende 1988 400.000 Menschen Angola verlassen, zwischen 1,5 und 3,5 Millionen befanden sich in Angola auf der Flucht, vgl. Richter (Anm. 1), S. 26.

3

Als Folge der indirekten ökonomischen sowie direkten militärischen Interventionen Südaf rikas flohen von 1981 bis 1988 1,2 Millionen Mosambikaner ins Exil, während rund 3,5 Millionen Menschen Binnenvertriebene (internally displaced persons) waren, vgl. Richter (Anm. 1), S. 20. 
schen Nachbarländern zu verhindern, haben zu Flucht und Vertreibung hunderttausender Menschen geführt. ${ }^{4}$

Neben der Destabilisierungspolitik war die Politik der Apartheidherrschaft auf die gewaltsame Vertreibung der schwarzen Bevölkerung aus Südafrika gerichtet. Zwischen 1960 und 1980 waren es z.B. ca. 3,5 Millionen Südafrikaner, die von der Apartheidpolitik innerhalb Südafrikas vertrieben worden waren. ${ }^{5}$ Zudem wurden südafrikanische Flüchtlinge, die in Nachbarländer wie Botswana, Lesotho oder Mosambik geflohen waren, von Agenten oder Handlangern des Apartheidregimes getötet. ${ }^{6}$

\section{Der Aliens Control Act und die Wende in Südafrikas Flüchtlingspolitik}

Das Apartheidregime kannte kaum gesicherte Grundlagen für die Behandlung von Asylgesuchen. Es waren lediglich die weißen Europäer, die nach der Unabhängigkeit von Mosambik, Angola und dem ehemaligen Rhodesien in Südafrika Schutz suchten und mit offenen Armen empfangen wurden. Dagegen beruhte die Einwanderungspolitik des Apartheidregimes für Nichtweiße auf dem Prinzip von "control and deportation". Die schwarzen Flüchtlinge aus benachbarten Ländern, beispielsweise Mosambikaner, galten in Südafrika als illegale Immigranten. Bis zur Verabschiedung des hier zu behandelnden Refugees Act von 1998 bediente man sich des 1991 verabschiedeten, mehrmals ergänzten und als "draconian Apartheid throwback" sowie "apartheids last act" ${ }^{7}$ charakterisierten Aliens Control Act 1991. Wie schon der Name sagt, war der Aliens Control Act alles andere als ein geeignetes Instrumentarium für die Bestimmung des Flüchtlingsstatus. In ihm sucht man den Ausdruck Flüchtling oder Asyl vergeblich. Sie wurden als "probihited persons" betrachtet und auch dementsprechend behandelt.

Die Bereitschaft Südafrikas, mit dem UNHCR formellen Kontakt aufzunehmen, dem bis dahin jeglicher Zugang zu dem Land verwehrt worden war, führte 1991 zum Wendepunkt in der Flüchtlingspolitik Südafrikas. In jenem Jahr traf die damalige Regierung de Klerk eine Vereinbarung mit dem UNHCR, das sog. Memorandum of Understanding, wonach die

S. Ostheimer, Andrea, Südafrikas Beziehungen zu Mozambique und Angola, in: Ulrike Schuhmacher (Hrsg.), Das Neue Südaf rika. Das Ende einer Mlusion?, 1997, S. 315-337 (317); UNHCR (Hrsg.), Zur Lage der Flüchtlinge in der Welt, 1995/96, S. 162.

5 U.S. Committee for Refugees (USCR), Country Information: South Africa, http://www. refugees.org/world/countryrpt/africa/past/south_africa.htm.

6 Vgl. UNHCR, (Anm. 4), S.100.

7 Vgl. Crush, Jonathan, Immigration, Human Rights and the Constitution in: ders., (Hrsg.), Beyond Control: Immigration and Human Rights in a Democratic South Africa, 1998, S. 1-17 (1). 
vom Apartheidregime vertriebenen und im Exil lebenden ca. 40.000 Südafrikaner zurückkehren durften.

Die erste Verpflichtung Südafrikas in Flüchtlingsangelegenheiten markierte das sog. Tripartite Agreement ${ }^{8}$ zwischen Südafrika, Mosambik und UNHCR vom September 1993, mit dessen Hilfe 35000 mosambikanische Flüchtlinge repatriiert werden sollten. Außerdem schloß Südafrika mit dem UNHCR das sog. Basic Agreement vom 6. September 1993. ${ }^{9}$ Das Abkommen sah die Errichtung eines UNHCR-Büros mit der Gewährleistung gewisser diplomatischer Privilegien vor. Zudem verpflichtete sich die Regierung, ein Verfahren zur Festlegung des Flüchtlingsstatus einzuführen. Daraufhin wurde im Innenministerium eine Abteilung für Flüchtlingsfragen eingerichtet.

Die neu eingerichtete Abteilung des Department of Home Affairs hat danach in Rücksprache mit dem UNHCR ein Verfahren für die Festlegung der Flüchtlingseigenschaft vorgelegt. Auf der Basis dieser Vorlage erließ das Innenministerium Richtlinien, die bei der Entscheidung über den Flüchtlingsstatus eingehalten werden sollten. In dem ad hoc-Asylverfahrensrecht wurde somit die Definition des internationalen Flüchtlingsrechts übernommen. ${ }^{10}$ Es wurde zum ersten Mal bei der Entscheidung über den Status mosambikanischer Flüchtlinge angewandt.

Mit dem Ende der Apartheidära und der Errichtung des demokratischen Systems 1994 in Südafrika hat sich die Flüchtlingsfrage grundlegend geändert. Bereits in der am 27. April 1994 in Kraft getretenen Interimverfassung von $1993^{11}$ bekannte sich Südafrika ausdrücklich dazu, die völkerrechtlichen Vereinbarungen zu unterschreiben, dann nach deren Ratifikation ins nationale Recht umzusetzen. ${ }^{12}$ Unmittelbar nach ihrer Machtübernahme 1994 zeigte die neue Regierung unter Nelson Mandela ihre Bereitschaft, den internationalen Vereinbarungen über Menschenrechtsfragen beitreten und sie national umsetzen zu wollen. So unterschrieb Mandela bereits am 3. Oktober 1994 während seines ersten Besuchs nach

The Tripartite Agreement between the Government of the Republic of South Africa, the Government of the Republic Mozambique and the UNHCR for the Voluntary Repatriation of Refugees from the Republic of South Africa, s. UNHCR (Hrsg.), REFWORLD, 1997.

Basic Agreement between the Government of the Republic of South Africa and the United Nations Higher Commission Concerning the Presence, Role, Legal Status of the UNHCR and its Personnel in the Republic of South Africa, vgl. UNHCR, REFWORLD (Anm. 8).

Klaaren, Jonathan, The History, Present Shape, and Administrative Future of Procedures for Refugee Status Determination, 29.3.1998, http://www.law.wits.ac.za/docs/lhrconf.htm.

Interim Constitution, Act No.200/1993, Art. 82(1) und Art. 231 (3), http://www. constitution.org.za/zip.html.

12

Ausführlich hierfür: de Wet, Erika, The Place of Public International Law in the New South African Constitutional Order: With Special Reference to International Human Rights and Humanitarian Law, in: Recht in Afrika, 2/98, S. 207-234. 
seiner Freilassung bei der Generalversammlung der Vereinten Nationen die beiden internationalen Pakte über bürgerliche und politische Rechte und über wirtschaftliche, soziale und kulturelle Rechte vom 16. Dezember $1966 .^{13}$

Einen entscheidenden Schritt für den Schutz der Flüchtlinge machte Südafrika, als es im Dezember 1995 die OAU-Konvention über spezifische Aspekte des Flüchtlingsproblems in Afrika von 1969 unterschrieb und im Januar 1996 der Genfer Konvention über die Rechtsstellung der Flüchtlinge von 1951, und dem Protokoll aus dem Jahre 1967 beitrat. $^{14}$ Im Vergleich zu anderen internationalen Verträgen in Menschenrechtsfragen, die von Südafrika unterschrieben worden waren ${ }^{15}$ und immer noch auf ihre Ratifizierung warten, wurde der Ausarbeitung des Flüchtlingsgesetzes absoluter Vorrang eingeräumt. Hierfür sollte die Bereitschaft Südafrikas, seinen vertraglichen Verpflichtungen nachzukommen, nicht der alleinige und ausschlaggebende Grund gewesen sein.

Mit dem Machtwechsel am Kap hat Südafrika den Ruf des fluchtverursachenden Landes, ("refugee producing country") abgelegt, und wurde plötzlich zu einem der bedeutendsten Aufnahmeländer für Flüchtlinge in Afrika ${ }^{16}$. Diese Anziehungskraft geht aber nicht auf die rechtliche Ausgestaltung der Flüchtlingsauf nahme zurück, sondern die Wahl Südafrikas als Fluchtziel läßt sich besser anhand der sog. "Pull-Faktoren" erklären. Als Fluchtmotivation unterscheiden die Migrationswissenschaftler zwischen Push- und Pull-Faktoren. Als primäre Auslöser für den Aufbruch aus der angestammten Heimat gelten danach Push-Faktoren (Schubkräfte) wie politische Verfolgung, Hunger, Kriege und Bürgerkriege, Umweltzerstörungen und ethnische Konflikte. Als Pull-Faktoren (Sogkräfte) kommen Anziehungspunkte wie Wohlstand und Stadtkultur bei der Wahl eines Zufluchtsorts in Betracht. 17

Unter Berücksichtigung der Pull-Faktoren erklärt sich die Anziehungskraft Südafrikas größtenteils anhand von folgenden Faktoren: Der erste und wichtigste Faktor ist wirtschaftlicher Natur. Südafrika gilt als ein "entwickeltes" Land innerhalb Afrikas südlich der

S. Liebenberg, Sandra, The International Convention on Economic, Social and Cultural Rights and its Implications for South Africa, in: South African Journal of Human Rights, vol. 11, Part 3, 1995, S. 359/378 (359).

Vgl. USCR, (Anm. 5).

S. die von Südafrika ratifizierten Menschenrechtsverträge, in: Human Rights Treaties Ratified by South Africa, http://www.law.wits.ac.za/docs/ratlist.html.

Bis Januar 1998 stellten 38.144 Flüchtlinge Asylantrag in Südafrika, davon waren 5.944 anerkannte, 21.704 unbearbeitete und 9.428 abgelehnte Anträge, vgl. Handmaker, Jeff, Refugees, Migrants and Policy Development: A Critical Look at the South African 1997 Draft Green Paper on Intemational Migration, http://www.lhr. org.za/refugee/green.htm.

S. Loescher, Gil, Beyond Charity: International Cooperation and Global Refugee Crisis, 1993, S. $16 \mathrm{f}$. 
Sahara, wenngleich bei der Verteilung des Pro-Kopf-Einkommens große Unterschiede nicht zu bestreiten sind. ${ }^{18}$ Der Vorsprung der südafrikanischen Wirtschaft wird besonders deutlich am Bruttosozialprodukt (BSP) der SADC (Southern African Development Community: Angola, Botswana, Lesotho, Malawi, Mauritius, Mosambik, Namibia, Sambia, Simbabwe, Swasiland und Tansania): Mit einem BSP, das fünfmal höher als das aller anderen SADC-Mitglieder ist, wird Südafrika zum „Wirtschaftsriesen" in der Region. ${ }^{19}$ Daher sind es hauptsächlich ökonomische Motive, die viele Menschen, die in ihrer Heimat keine Zukunft mehr sehen, z. B. in benachbarten Ländern wie Mosambik, dazu bewegen, in Südafrika Arbeit zu suchen. Für sie ist Südafrika das Land, wo Milch und Honig fließen ("Land of milk and honey"). ${ }^{20}$ Darüber hinaus spielt die auf demokratischer und rechtsstaatlicher Basis beruhende Verfassung Südafrikas bei der Wahl der Flüchtlinge eine bedeutende Rolle. Denn die am 4. Februar 1997 verabschiedete endgültige Verfassung Südafrikas wird wegen ihrer Menschenrechtsgarantie (Bill of Rights im zweiten Kapitel der Verfassung) - schon in der Übergangsverfassung von 1993 befaßten sich 28 Artikel (Art. 7-35) mit Grundrechten, die internationalen Anforderungen entsprachen - vielfach als die „liberalste Verfassung der Welt" gepriesen. ${ }^{21}$ Zudem gilt das Verfassungsgericht des Landes auch als unabhängig. ${ }^{22}$

Nicht zu übersehen ist ebenfalls die Machtstellung Südafrikas im Kontinent, die für die Attraktivität des Landes nicht unbedeutend ist. Bei vielen Kommentatoren gilt Südafrika heute schon als die unbestrittene führende Macht in Afrika. ${ }^{23}$

Die in den letzten Jahren immer restriktiver werdende Flüchtlingspolitik in Europa und Nordamerika hat ebenfalls dazu beigetragen, daß viele Flüchtlinge ihr Heil nun in Südafrika suchen. $^{24}$

In dem Bericht des Entwicklungsprogramms der UN (UNDP), Human Development Report 1997, wurde Südafrika als "medium developed" bezeichnet. Danach nimmt Südafrika zwar Rang 90 in der Welt ein, steht aber vor allen Ländern Afrikas, mit Ausnahme von Algerien und Tunesien, an erster Stelle, vgl. Handmaker, (Anm. 16).

Vgl. Handmaker, (Anm. 16).

Vgl. von Lucius, Robert, Fünfzig Jahre Menschenrechtserklärung: Erblast der Rassendiskriminierung, in: FAZ vom 27.11.1998, S. 6; Devenish, George E., The Republic of South Africa Constitutional Act, 1996: A Commentary on its Content and on the Certification Process Conducted by the Constitutional Court, in: Jahrbuch für Afrikanisches Recht, 9/1997. S. 3-31 (12). Verfahren gegen Wahl in Südafrika, in: FAZ vom 06.04.1999, S. 7. Eine Bilanz nach zehn Jahren politischer Veränderungen seit dem Ende des Kalten Krieges, in: Aus Politik und Zeitgeschichte, B 21/98, 15. Mai 1998, S. 3-15 (11). 
Aufgrund der oben aufgeführten Pull-Faktoren erlebt Südafrika seit der Abschaffung der Apartheid einen Zustrom von Menschen, nicht nur aus Afrika, sondern auch aus Südamerika $^{25}$, Asien ${ }^{26}$ und sogar aus Europa. ${ }^{27}$ Das von dem Green Paper Task Team am 30 Mai 1997 vorgelegte Draft Green Paper on International Migration ${ }^{28}$ teilt die heute nach Südafrika strömenden Menschen in drei Kategorien ein: An erster Stelle kommen Immigranten, die sich in Südafrika permanent niederlassen wollen. Unter die zweite Kategorie fallen Flüchtlinge, die vor der Verfolgung in ihren Heimatländen entfliehen und in Südafrika Asyl suchen, ${ }^{29}$ während die illegalen Einwanderer zu der dritten Gruppe gehören. Wenngleich sich präzise Angaben über ihre Zahl schwer ermitteln lassen, halten sich schätzungsweise zwischen 5-8 Millionen "illegale" Immigranten - ein Fünftel der Einwohner des Landes -, die Mehrheit davon aus Mosambik und Simbabwe, in Südafrika auf. ${ }^{30}$ Die Illegalen stammen nicht nur aus benachbarten Staaten. Seit Südafrika mit China diplomatische Beziehung pflegt, verzeichnet man eine steigende Anzahl von Illegalen aus dem Reich der Mitte. ${ }^{31}$ Nach Schätzungen des Task Team on International Migration würde die Rückführung dieser Illegalen in ihre Heimatländer - Südafrika deportiert ca. 160.000200.000 Ausländer jährlich - zwischen fünfundzwanzig und dreißig Jahre in Anspruch nehmen. $^{32}$

Zwischen September 1996 und Mai 1997 zählte man 4000 argentinische Flüchtlinge in Südaf rika, vgl. Williams, Lionel, Thousands Seek Asylum in South Africa, in: Panafrican News Agency (PANA) vom 15. Mai 1997, http://www.africanew.org.

Unter anderem Flüchtlinge aus Bangladesch, Indien und Pakistan, vgl. James, Wilmot, Migration Regulations should be More Flexible, in: Cape Times von 11. Juni 1997, http://www. quennsu.ca/publications/artic11.htm.

Es sind u.a. Flüchtlinge aus Bulgarien und aus dem ehemaligen Jugoslawien, s. UNHCR, (Anm. 4), S. 206; USCR, (Anm. 5).

Draft Green Paper on Intermational Migration, Government Gazette, vol. 383, No. 18033, http://www.polity.org.za/ govdocs/green_paper.

Im Vergleich zu den "Illegalen", ist die Anzahl der Flüchtlinge wesentlich geringer. 1997 haben in Durchschnitt 1000 Flüchtlinge pro Monat einen Asylantrag gestellt, während ihre Zahl 1999 sich auf 1500 Anträge erhöht hat, vgl. Williams, (Anm. 25); Migration News vol. 6, No. 4, April 99, http://migration.ucdavis.edu.

Greybe, David, Policy Revamp on Migrants: White Paper Focuses on Meeting Skills Needs, in: Business Day vom 7.4.1999, http://www.bday.co.za/99/ 0407/news/news1.htm; Daley, Suzanne, New South Africa Shuts the Door on its Neighbours, in: The New York Times vom 19.10.1998, http://www.nytimes.com.

S. Hartely, Wyndham, Chinese Mlegal Aliens Start Concem, in: Business Day vom 19.4.1999, http://www.bday.co.za/99/ 0419/news/news10.htm.

Vgl. Task Team on Intemational Migration, White Paper on Intemational Migration: Presented to the Minister of Home Affairs, March 31, 1999, http://www.polity.org.za/gov.white_papers/ migration.html. 
Wie man der obigen Zahl entnehmen kann, stellt der wachsende Migrationsstrom mit Ziel Südafrika ein erhebliches Problem für das Land dar. Illegale Grenzübertritte - trotz der Sicherung der Grenzen mit Elektrozaun und Einschaltung der Spezialeinheit der Polizei, bewaffneter Zivilisten und der Armee - konnte man nicht eindämmen. Wie Südafrika überfordert wurde und wie ernst die Lage ist, zeigt die Bitte Südafrikas an Deutschland ${ }^{33}$, ihm Ratschläge für eine bessere Kontrolle an den Grenzen zu erteilen. Daraufhin traf eine Delegation aus Sachsen in Südafrika ein. Nachdem die Delegation sich vor Ort ein Bild gemacht hatte, traf man eine Vereinbarung, bei Grenzkontrollen zusammenzuarbeiten. ${ }^{34}$ Die Ankunft vieler Ausländer in Südafrika, unabhängig davon, ob man Flüchtling, Immigrant oder "Illegaler" ist, ruft bei den Einheimischen negative Reaktionen hervor. Dies ist an erster Stelle auf die eigenen Probleme im Lande zurückzuführen. Trotz der ,positiven Diskriminierung" ${ }^{35}$ des Employment Equity Bill, sind 40\% der südafrikanischen Bevölkerung ohne Arbeit. ${ }^{36}$ Die Regierung steht unter beträchtlichem Erwartungsdruck insbesondere der schwarzen Bevölkerung. Alles soll besser werden, und zwar sofort. Bisher hat die Regierung die in sie gesetzten Erwartungen jedoch nur zum Teil erfüllen können. In dieser Situation werden Ausländer zum Sündenbock gemacht. Nach der verbreiteten Meinung der Medien, der Politiker ${ }^{37}$ und der Bevölkerung nähmen Ausländer die Arbeitsplätze weg, sie brächten Krankheiten ${ }^{38}$ mit und seien kriminell. ${ }^{39}$ Dadurch hat sich in der Bevölkerung eine wachsende Fremdenfeindlichkeit entwickelt. Wie diesbezügliche Untersuchungen des

Anfang des Jahres traf eine südafrikanische Delegation in Deutschland ein, um bei der Bekämpfung der Kriminalität und der Kontrolle der illegalen Grenzübertritte von der Erfahrung Deutschlands zu lernen; vgl. Dludlu, John, Northern Province Seeks German Advice on Aliens, in: Business Day vom 8. März 1999, http://www.bday.co.za/99/0308/news/ nwes1.htm.

Makgotho, Selby, Germans set to help catch Northern Province's immigrants, in: African Eye News Service vom 3.5.1999, http://www.cis.org.

35 Schwertfeger, Bärbel, Kalifornien am Kap, in: UNICUM, 2. Jg. Nr. 3, Mai/Juni 1999, S. $32-33$ ( 33).

36 FAZ vom 11..3.99, S. 2; Behrens, Gerd, Südafrika am Ende der Ära Mandela: Versöhnen im Schatten der Gewalt, in: Süddeutsche Zeitung vom 27.5.1999, S. 3; U.N. Predicts Southem Africa Refugee Crisis, Reuters (Johannesburg) vom 4.3.1999, http://www.cis.org.

Allen voran der Innenminister Mangosuthu Buthelzi, der öffentlich behauptet, daß „die Einwanderer für Südafrika eine ernsthafte Bedrohung darstellen", vgl. Handmaker, (Anm. 16), Fußnote 7.

Ausländer werden für die Verbreitung von AIDS verantwortlich gemacht. Südafrika hat die weltweit höchste AIDS-Infektionsrate, jeder Vierte hat das Virus in sich, vgl. Behrens, (Anm. 36).

Nach Angaben der South African Police Service (SAPS) beträgt die Kriminalitätsrate der Ausländer vierzehn Prozent. Zu den von Ausländem begangenen Straftaten gehören Geldwäsche, Autodiebstahl und Waffenschmuggel, s. Africans Lured to South Africa find Racisim and Hostility, in: South African Press Agency (SAPA) vom 23.10.98, http://www.cis.org; Williams, Vincent, Green Paper Signals Break with Racist Past, in: Crossings vol. 1, No. 1, September 1997, http://www.queensu.ca/publications/artic29.htm; Human Rights Watch, "Prohibited Persons": Abuse of Undocumented Migrants, Asylum-Seekers, and Refugees in South Africa, March 1998, http://www.hrw.org/reports98/sareport; Repatriating Aliens Costs SA R200 Million a Year, in: SAPA vom 18. Juni 1998, http://www.cis.org. 
Instituts für Demokratie in Südafrika verdeutlichen, sind die Einheimischen in Südafrika wie in keinem Land der Welt strikt gegen die Einwanderung: 25\% der Bevölkerung ist für ein totales Einwanderungsverbot, während $45 \%$ für eine strenge Begrenzung des Zuzuges der Ausländer plädieren. ${ }^{40}$ Der kürzlich veröffentlichte Bericht der Menschenrechtskommission Südafrikas ${ }^{41}$ macht deutlich, daß die Xenophobie und der damit zusammenhängende Rassismus in Südafrika alarmierend sind. Mehrfach wurden Flüchtlinge angegriffen, umgebracht - allein in zwei Jahren (1997-98) fielen dreißig Flüchtlinge ausländerfeindlichen Übergriffen zum Opfer ${ }^{42}$ - und von der Polizei mißhandelt. Daher wundert es kaum, wenn Südafrika derzeit von einigen Kommentatoren als eines der ausländerfeindlichsten Länder in der Welt abgestempelt wird. ${ }^{43}$ Es klingt zwar ironisch, aber es gilt nach wie vor der althergebrachte Apartheidmaßstab, nämlich die Hautfarbe. Je heller die Farbe eines Flüchtlings - Immigranten aus Europa und Nordamerika sind willkommen -, um so geringer ist die Gefahr, angegriffen und abgelehnt zu werden. ${ }^{44}$ Wie zu Apartheidzeiten sind es viele schwarze Südafrikaner, die aufgrund ihrer angeblich "zu dunklen" Hautfarbe regelmäßig von der Polizei verhaftet und in die Nachbarländer abgeschoben werden.

Deshalb liegt es auf der Hand, daß die Regierung durch den Erlaß eines Asylgesetzes dem ansteigenden Zuzug von Ausländern entgegenwirken, die Zuwanderung ${ }^{45}$ regulieren und den eigenen Bürgern das Gefühl geben möchte, mit der Ausarbeitung eines Gesetzes ${ }^{46}$ die Kontrolle darüber zu haben, wie, wie vielen und welchen Menschen die Einreise und der Aufenthalt im Lande erlaubt werden sollen.

40

41

42

43

44

45

46

Vgl. UNHCR (Hrsg.), Refugees, vol. 2, 115/1999, S. 27.

South African Human Rights Commission, Report into the Arrest and Detention of Suspected Undocumented Migrants, 19 March 1999, http://www.niza.nl/uk/hrc/hrcreport.htm.

S. Reuters (Johannesburg) vom 4.3.1999, http://www.queensu.ca/samp/news.htm.

"... South Africans are among the world's most xenophobic people", s. Daley (Anm. 30); Jonathan Crush und Robert Mattes, Xenophobia: Hostility ,Growing Alarmingly', in: Crossings, vol. 2, No. 3, Oktober 1998, http://www.queensu.ca/publications/artic31.htm.

Vgl. auch Brandt, Hans, Wer zu schwarz ist, lebt gefährlich: Südafrikas Ausländerpolizei macht Jagd auf illegale Einwanderer / Willkür, Korruption und Rassismus grassieren, in: Frankfurter Rundschau vom 15.4.1999, S. 3.

In dem Zusammenhang sei es erwähnt, $\mathrm{da} B$ das bereits erwähnte Task Team on International Migration seine endgültige Arbeit abgeschlossen und das Weißbuch zur Migrationsfragen dem Innenminister vorgelegt hat. In dem bereits am 31. März 1999 vom Kabinett gebilligten Weißbuch empfahl das Team die Schaffung eines besonderen Sicherheitsservice, separater Hafteinrichtungen für illegale Einwanderer, die Ermächtigung des Immigration Service, als illegal verdächtigte Ausländer ohne gerichtlichen Haftbefehl für 48 Stunden festzuhalten und strenge Strafen für jene Arbeitgeber, die illegale Arbeitskräfte beschäftigen sowie die Schaffung eines Immigration Court, vgl. Anm. 32.

Zu den anderen möglichen Beweggründen s. die Thesen von Sinclair, Marion Ryan, International Refugee Treaties and their Implications for the South African State, Centre for Southern African Studies, 1998, S. 7 ff. 
Auf der anderen Seite steht der ANC in der Schuld vieler benachbarter Länder in Afrika, die ihm während der Apartheidherrschaft Zuflucht gewährten und die Widerstandsbewegung förderten. ${ }^{47}$ Insbesondere spielte die "Befreiungsallianz" der damaligen Frontstaaten (Angola, Botswana, Lesotho, Malawi, Mosambik, Namibia nach der Unabhängigkeit, Sambia, Simbabwe, Swasiland und Tansania) durch die Unterstützung des Kampfs gegen das Apartheidsystem eine entscheidende Rolle. Sie umfaßte u.a. die Asylgewährung an die Befreiungskämpfer des ANC und PAC. So genossen die ANC-Kämpfer als sog. Freiheitskämpfer (freedom fighters) ${ }^{48}$ im Einklang mit der OAU-Flüchtlingskonvention Schutz und Unterstützung, verbunden mit dem Flüchtlingsstatus. Beispielsweise erhielten sie in Tansania eine privilegierte Aufnahme und wurden in Trainingslagern für den Kampf gegen das Apartheidregime ausgebildet. ${ }^{49}$ Die damaligen Befreiungskämpfer und die heutigen Politiker Südafrikas wissen auch diesen Beitrag zu schätzen, wie ihren Bekenntnissen zu entnehmen ist: "Tansania, Simbabwe, Botswana and other countries to which we fled in the 1960s did not call us illegal aliens. They said,we are going to support our brothers and sisters from South Africa so that they can go home". ${ }^{50}$ Diese Erwartungshaltung vieler Menschen aus benachbarten und anderen Ländern Afrikas gegenüber der vom ANC geführten Regierung wird durch die folgende Aussage der Tochter des ehemaligen Präsidenten Sambias, Kaunda, unmißverständlich zum Ausdruck gebracht. Nachdem sie im Zusammenhang mit der Verhaftung ihres Vaters, dem nun seine Staatsbürgerschaft aberkannt wurde, in Südafrika Ende 1997 Asyl beantragt hatte und ihr Ersuchen als "offensichtlich unbegründet" abgelehnt wurde - trotz ihrer schriftlichen Bitte an Nelson Mandela -, äußerte sie ihre Enttäuschung in Anspielung auf die Vergangenheit: Sie sei zwar nicht verbittert, aber sie vergesse nicht, daß ihre Familie Gastgeber der Abendgesellschaften einiger Spitzenpolitiker Südafrikas gewesen sei. Als ihr Vater Präsident war, da sei sein Haus offen für seine Freunde aus Südafrika gewesen. ${ }^{51}$

Den Weg für die Ausarbeitung eines nationalen Flüchtlingsgesetzes ebnete das Memorandum of Agreements vom Juli 1996 zwischen Südafrika und dem UNHCR - und dies, obwohl eine mit dieser Aufgabe betraute Abteilung im Innenministerium sich schon seit 1994 mit einer Entwurfsarbeit eines Flüchtlingsgesetzes befaßt und eine erste Fassung der

Vgl. Ostheimer, (Anm. 4), S. 327.

S. hierfür Hofmann, Rainer, Zur Flüchtlingsproblematik in Afrika, in: Jahrbuch für Afrikanisches Recht, 3/1982, S. 105-134 (120).

49 Vgl. Weigang, Silke, Flüchtlingsansiedlungen in Tansania als Beitrag zur regionalen Entwicklung, in: Afrika Spectrum 31 (1996) 2, S. 137-160 (141).

50

Thambo Mbeki, zitiert in Hunt, Lee Anne de la, Refugees and Immigration Law in South Africa, in: Jonathan Crush (Hrsg.), Beyond Control: Immigration and Human Rights in a Democratic South Africa, 1989, S. 123-140 (124).

51 Vgl. Bezuidenout, Jessica, South Africa Rejects Kaunda's Daugther, in: Sunday Times vom 11. April 1999, http://www.suntimes.co.za/1999/04/11/news/guateng/ njhb11.htm. 
Refugee Bill schon 1994 aufgestellt hatte -, wonach Südafrika sich zur Ausarbeitung einer vereinheitlichenden Regelung der Flüchtlingsfragen verpflichtet hatte.

Die sich auf die Vorarbeit der genannten Abteilung stützenden Entwurfsarbeiten des vom Innenminister berufenen Task Team, die sog. Draft Refugee White Paper ${ }^{52}$ und die Draft Refugee Bill ${ }^{53}$, wurden am 18. Juni 1998 fertiggestellt, woraufhin sie mit der Veröffentlichung im Amtsblatt am 20. Juni 1998 der Öffentlichkeit präsentiert und zur Diskussion gestellt wurden. Mit Hilfe des Internets warb man für Änderungs- bzw. Verbesserungsvorschläge, die bis 20. Juli 1998 eintreffen sollten. Parallel dazu legte das vom Innenminister im Jahre 1996 eingesetzte Green Paper Task Team on International Migration dem Minister des Innern am 30. Mai 1997 das oben genannte Draft Paper on International Migration vor. In dem Draft Paper widmete das Team ein Kapitel der Flüchtlingsfrage und empfahl, die Einwanderungs- und Flüchtlingsfragen separat zu regeln. Es unterstrich zudem auch die Notwendigkeit eines eigenständigen Flüchtlingsgesetzes. So forderte es in seiner Empfehlung: "we believe that there is an urgency to enact legislation to establish a formal system of refugee protection in South Africa. ... Refugee protection unlike immigration, is fundamentally a matter of implementing international legal obligation in domestic law". ${ }^{54}$

Nachdem zahlreiche Ergänzungs- und Verbesserungsvorschläge von sechzehn Organisationen (u.a. von UNHCR, Human Rights Watch, Lawyers for Human Rights, South African Human Rights Commission und Amnesty International) eingegangen waren ${ }^{55}$, wurde die Refugee Bill fertiggestellt und am 5. November 1998 in die Nationalversammlung eingebracht. ${ }^{56}$ Der vom Parlament am 20. November 1998 verabschiedete Refugees Act No. 130 trat nach seiner Verkündung durch den Präsidenten und mit der Veröffentlichung im Gesetzblatt am 2. Dezember 1998 in $\mathrm{Kraft}^{57}$.

\section{Die Bestimmungen des Refugees Act}

Der Refugees Act besteht aus sechs Kapiteln und einundvierzig Artikeln. Während Kapitel eins den materiellen Teil - Begriffserklärungen, Interpretation und Flüchtlingsdefinition enthält, befaßt sich Kapitel zwei mit institutionellen Fragen wie der Errichtung und den

55 Vgl. http://www.polity.org.za/govdocs/bills/1998/b135-98.pdf.

56 The Draft Refugee Bill, No. 135, http://www.polity.org.za/govdocs/bills/1998/index.htm. 
Kompetenzen der zuständigen Organe für die Umsetzung des Gesetzes. Die Kapitel 3-5 widmen sich den Verfahrensfragen. Kapitel sechs enthält schließlich allgemeine Vorschriften.

\subsection{Voraussetzungen der Asylgewährung}

Die Voraussetzungen, unter denen einem Ausländer aufgrund seiner Flüchtlingseigenschaft Asyl gewährt wird, bestimmt das Gesetz in Art. 3 a-c.

\subsubsection{Der Flüchtlingsbegriff}

Artikel 3 des Refugees Act bestimmt den Flüchtlingsbegriff für die Zwecke des Refugees Act. Dabei lehnt sich Artikel 3 a weitgehend an die Formulierung von Art. 1 Abs. 2 der Genfer Flüchtlingskonvention von 1951 an. ${ }^{58}$ Wie der Vergleich der beiden Definitionen verdeutlicht, ist die einzige in dem Refugees Act als neu zu bezeichnende Fluchteigenschaft die Zugehörigkeit zu einem Stamm. Danach gilt die Verfolgung wegen Zugehörigkeit zu einem bestimmten Stamm als Fluchtgrund. Gerade in Afrika, wo die internen Konflikte mit erbitterten Feindseligkeiten zwischen Volksgruppen, Stämmen und Gemeinschaften einhergehen und zur Flucht einer Volksgruppe führen können - siehe Hutu und Tutsi in Zentralafrika -, entspricht die Aufnahme eines solchen Fluchtgrundes in den Katalog der Flüchtlingseigenschaften der afrikanischen Wirklichkeit.

Eine weitere Neuheit bei der Definition durch den Refugees Act ist die Bestimmung des Kreises der Personen, die unter "soziale Gruppe" zu subsumieren sind. Im internationalen Flüchtlingsrecht herrscht keine Einigkeit darüber, welche Kategorien von Personen als Angehörige der "bestimmten sozialen Gruppe" angesehen werden können. Nach der Rechtsprechung einiger Staaten (Deutschland, Dänemark, Kanada und die Niederlande) wurden Menschen aufgrund ihres Geschlechts und ihrer sexuellen Orientierung unter diesem Gesichtspunkt als Flüchtlinge anerkannt ${ }^{59}$. So sprach das Ansbacher Verwaltungsgericht einer Rumänin Asyl zu, weil sie von Angehörigen der Staatssicherheit in ihrer Heimat sexuell mißhandelt worden war. ${ }^{60}$ Ähnlich gewährte der 9. Senat des Bundesverwaltungs-

Nach der Genfer Konvention über die Rechtsstellung der Flüchtlinge findet der Ausdruck "Flüchtling" auf jede Person Anwendung, die sich aus der begründeten Furcht vor Verfolgung wegen ihrer Rasse, Religion, Nationalität, Zugehörigkeit zu einer bestimmten sozialen Gruppe oder wegen ihrer politischen Überzeugung außerhalb des Landes befindet, und den Schutz dieses Landes nicht in Anspruch nehmen kann oder wegen dieser Befürchtungen nicht in Anspruch nehmen will", vgl. UNTS, vol. 189, S. 151 ff; deutscher Text, BGBl 1953 II, S. 560 ff.

Carlier, Jean-Yves (Hrsg.), Who is a Refugee? A Comparative Case Study, 1997, S. 713.

60

Hullmann, Klaus, Germany, in: Jean-Yves Calier, (Anm. 58), S. 225-291 (284). 
gerichts einem Iraner aufgrund seiner "irreversiblen, schicksalhaften homosexuellen Prägung" Asyl. ${ }^{61}$ Im Sinne des Art. 1 (xxi) des Refugees Act umfaßt eine "soziale Gruppe": "... a group of persons of particular gender, sexual orientation, disability, class or caste".

Der Refugees Act beschränkt sich nicht nur auf die Übernahme der Definition der Genfer Flüchtlingskonvention, sondern er lehnt sich auch weitgehend an die Definition der OAUKonvention über spezifische Aspekte des Flüchtlingsproblems in Afrika von 1969 an. ${ }^{62}$ Die Begriffsbestimmung des Artikels 3 (b) ist fast die Kopie der Definition des Art. I (2) der OAU-Flüchtlingskonvention. Danach erkennt Südafrika auch jede Person als Flüchtling an, die "infolge einer von außen kommenden Aggression, ausländischer Besetzung, Fremdherrschaft oder schwerwiegender Störungen ("seriously disturbung or disrupting") der öffentlichen Sicherheit und Ordnung in ihrem Herkunftsland oder dem Land, dessen Staatsangehörigkeit sie besitzt oder einem Teil derselben, gezwungen ist, ihren gewöhnlichen Aufenthaltsort zu verlassen, um Schutz woanders zu suchen".

Mit der Übernahme des im Vergleich zur Flüchtlingsdefinition der Genfer Flüchtlingskonvention von 1951 erheblich erweiterten Art. I (2) der OAU-Konvention dehnt der Refugees Act die Anwendbarkeit seiner Definition auf die sog. de-facto-Flüchtlinge ${ }^{63}$ aus. Trotz dieser Ausweitung der Flüchtlingseigenschaft auf subjektive Kriterien der OAU-Konvention wie Krieg und Besetzung, erkennt der Flüchtlingsbegriff des Refugees Act Personen, die wegen Natur- oder Hungerkatastrophen, aufgrund der allgemeinen schlechten wirtschaftlichen Lage ihr Land verlassen haben, nicht als Flüchtlinge an. Schon das Draft Refugee White Paper erteilte Flüchtlingen, die aufgrund von Armut, wirtschaftlicher Not und Umweltschäden ihren Heimatländern den Rücken gekehrt hatten, eine klare Absage: "It (die Regierung Südafrikas) does not agree that it is appropriate to consider as refugees, persons fleeing their countries of origin solely for reasons of poverty or other social, economic or environmental hardships". ${ }^{64}$ Diese Absicht wird in behördlichen Stellungnahmen des Landes betont, wonach Südafrika sich lediglich für den Schutz der echten Flüchtlinge stark machen will. So lehnt Innenminister Buthelezi die Aufwertung der Wirtschaftsflüchtlinge strikt ab: "We do not accept that economic migrants should be able to use the refugee determination process to obtain residence, employment ... or to avoid the consequences of the law concerning 'illegal migration'". 65

61

62

63

64

65

Vgl. BVerwGE 79, S. $143 \mathrm{ff}$.

Vgl. den Originaltext in: International Legal Materials 8/1969, S. 1288-1298. Die deutsche Übersetzung von Rainer Hofmann ist abgedruckt im Jahrbuch für Afrikanisches Recht, 3/1982, S. 243250.

Vgl. Hofmann, Zur Flüchtlingsproblematik, (Anm. 48), S.118 ff.

S. Draft Refugee White Paper, (Anm. 52).

White Paper Moots Legislation to End Abuse of South African Asylum System, in: South African Press Agency (SAPA) vom 18. Juni 1998, http://www.cis.org. 
Schließlich gelten nach Art. 3 (c) des Refugees Act die Familienangehörigen der in der Begleitung der nach Art. 3 a und b anzuerkennenden Personen als Flüchtlinge. Das Gesetz zählt zu den Familienangehörigen eines Asylsuchenden Gatte/in, unverheiratete Minderjährige unter 18 Jahre, oder irgend ein mittelloses, altes und schwaches Mitglied der Familie. ${ }^{66}$

\subsubsection{Die Ausschlußgründe der Flüchtlingseigenschaft}

Der Katalog der Ausschlußgründe einer Flüchtlingseigenschaft in Art. 4 des Refugees Act lehnt sich bei weitem an die Genfer Flüchtlingskonvention und OAU-Konvention an. Danach genießt eine Person den Schutz des Refugees Act nicht, wenn sie - erstens - ein Verbrechen gegen den Frieden, ein Kriegsverbrechen oder ein Verbrechen gegen die Menschlichkeit im Sinne eines der internationalen Vertragswerke, die sich mit einem solchen Verbrechen befassen, begangen hat. Diese Bestimmung entspricht weitgehend den Regelungen des Art. I Abs. 5 (a) der OAU-Konvention und des Art. 1 Abschnitt F (a) der Genfer Flüchtlingskonvention. ${ }^{67}$ Zweitens kommt eine Person nicht in den Genuß des Flüchtlingsschutzes, wenn sie ein nicht-politisches Verbrechen in Südaf rika begangen hat, das einen Freiheitsentzug nach sich ziehen kann. ${ }^{68}$ Diese von den oben genannten Vertragswerken abweichende Bestimmung ist bedenklich. Während sowohl nach der Genfer Flüchtlingskonvention als auch in der OAU-Konvention eine Person von dem Genuß des Flüchtlingsschutzes ausgeschlossen wird nur, wenn sie "... ein schweres nicht-politisches Verbrechen außerhalb des Aufnahmelandes begangen hat, bevor sie dort als Flüchtling aufgenommen wurde", reduziert der Refugees Act dies auf jedes Verbrechen, das eine Haftstrafe vorsieht. Schon der ursprüngliche Ausschlußgrund der Genfer Flüchtlingskonvention ist in Lehre und Praxis umstritten. ${ }^{69}$ Einigkeit besteht darüber, daß für die Anwendbarkeit der Bestimmung drei Voraussetzungen kumulativ erfüllt sein müssen: Erstens muß es sich um Delikte handeln, die der Asylsuchende "außerhalb" des Zufluchtslandes verübt hat, "bevor" er dort als Flüchtling Aufnahme fand. Zweitens muß es sich um ein "Verbrechen des gemeinen Rechts" (ein nicht-politisches Delikt) handeln. Drittens muß es sich um ein schweres Verbrechen handeln. ${ }^{70}$ Nach herrschender Meinung fallen unter ein schweres Verbrechen Straftaten, die u. a. gegen körperliche Integrität, Leben und Freiheit gerichtet sind. ${ }^{71} \mathrm{Da}$ viele Straftaten, für die in Südafrika Haftstrafen verhängt werden, nicht als schwere Ver-

Art. 3 (c) i. V. m. Art. 1 (ix) des Refugees Act.

Art. 4 Abs. 1( a) des Refugees Act.

Art. 4 Abs. 1(b) des Refugees Act.

Vgl. hierzu Grahl-Madsen, Atle, Status of Refugees, vol. I, 1966, S. 294 ff; Goodwin-Gill, Guy S., The Refugee in International Law, $1996^{2}$, S. 104 ff; Gotffried Köfner / Peter Nicolaus, Grundlagen des Asylrechts in der Bundesrepublik Deutschland, 1986, S. 324 ff; Kälin, Walter, Das Prinzip des Non-Refoulement, 1982, S. $126 \mathrm{ff}$.

S. Kälin, Walter, Grundriß des Asylverfahrens, 1990, S. $174 \mathrm{ff}$.

Goddwin-Gill, (Anm. 69), S. 105. 
brechen eingestuft werden können, ist diese Bestimmung während der Entwurfsarbeit von Menschenrechtsorganisationen, ${ }^{72}$ Kirche ${ }^{73}$ und $\mathrm{UNHCR}^{74}$ heftig kritisiert worden. Trotz dieser Bedenken und Verbesserungsvorschlägen setzte sich der Entwurf in seiner ursprünglichen Fassung durch, der von seinem Wortlaut her von der Genfer Flüchtlingskonvention abweicht.

Übereinstimmend mit den Vorgaben des Art. 1 Abschnitt F (c) der Genfer Konvention und des Art. I Abs. 5 (d) wird gemäß Art. 4 Abs. 1 (c) des Refugees Act einer Person der Schutz vor Verfolgung verweigert, wenn sie sich Handlungen zuschulden kommen ließ, die den Zielen und Grundsätzen der Vereinten Nationen oder der OAU zuwiderlaufen. Dieser bereits nach der Verabschiedung der OAU-Konvention als "unverständlich" und "überflüssig" ${ }^{75}$ kritisierte Ausschlußgrund wird in Art. 4 Abs. 2 des Refugees Act abgemildert, sofern die Handlung zur Ausübung im Völkerrecht anerkannter Menschenrechte dient. Dort heißt es: "... no exercise of human rights recognized under international law may be regarded as being contrary to the objects and principles of the United Nations Organization or the OAU".

Schließlich wird die betreffende Person nach Art. 4 Abs. 1 (d) des Refugees Act aus dem Schutz des Refugees Act ausgeschlossen, wenn sie bereits in irgendeinem anderen Land in den Genuß des Schutzes gekommen war. Dahinter steht der Gedanke, jenen den Zugang zu einem Asylverfahren zu verweigern, die während ihrer Flucht anderswo hätten Schutz finden können.

\subsection{Zuständigkeit und Anerkennungsverfahren}

\subsubsection{Zuständigkeit für die Anerkennung von Flüchtlingen}

Zuständig für die Umsetzung des Refugees Act und damit für die Behandlung von Asylsuchenden ist das Department of Home Affairs, an dessen Spitze der vom Innenminister zu

72

Vgl. für die Stellungnahmen von Human Rights Watch, South African Human Rights Commission, Lawyers for Human Rights, Submission to the Parlamentary Portfolio Committee on the Refugee Bill vom 13. Oktober 1998, http://www.pgm.za.

South African Catholic Bishops Conference, Submission to the Portfolio Committee on Home Affairs on the Refugee Bill (B135-98), Oktober 1998, http://www.pgm.za.

74 United Nations High Commissioner for Refugees, Draft Refugee Bill, 1998, Version Reviewed by the State Law Advisor, http://www.pgm.za. zur Entwicklung des humanitären Völkerrechts, in: VRÜ 3 (1970), S. 443-459 (453). 
ernennende Generaldirektor steht. Der Generaldirektor hat somit den allgemeinen Richtlinien und Anordnungen des Innenministers Folge zu leisten.

Die Struktur der Behörden, die sich unmittelbar mit Anerkennungsverfahren befassen, unterteilt das Gesetz in drei Instanzen: Das Amt für die Flüchtlingsaufnahme (Refugee Reception Office), den Ständigen Ausschuß für die Flüchtlingsangelegenheiten (Standing Commitee for Refugee Affairs) und den Berufungsausschuß (Refugee Appeal Board).

Der Refugees Act ermächtigt den Generaldirektor, zentrale Aufnahmeeinrichtungen für Asylbegehrende einzurichten. Jede Einrichtung sollte mindestens mit einem Beamten für Flüchtlingsaufnahme (Refugee Reception Officer) und einem Beamten für die Entscheidung über Asylanträge (Refugee Status Determination Officer) besetzt sein. Diese vom Generaldirektor zu ernennenden Beamten müssen entsprechend qualifiziert und im Besitz von Erfahrung und Fachkenntnissen in Flüchtlingsfragen sein. Während die Aufgabe des Beamten für die Flüchtlingsaufnahme in der Entgegennahme der Asylanträge liegt, entscheidet der zuständige Beamte über den Asylantrag, einschließlich der Feststellung, ob die Voraussetzungen einer Verfolgung im Sinne der Definition des Refugees Act vorliegen.

Ferner sieht der Refugees Act die Errichtung eines mit weitgehenden Kompetenzen ausgestatteten Ständigen Ausschusses für Flüchtlingsangelegenheiten vor. Die Mitglieder des Ausschusses werden vom Innenminister auf fünf Jahre ernannt. $\mathrm{Zu}$ den Aufgaben des als unabhängig konzipierten Ausschusses gehören u.a. die Ausarbeitung und Umsetzung des Anerkennungsverfahrens für Flüchtlinge. Darüber hinaus überwacht er die Arbeiten des Refugee Reception Officers. Er knüpft auch den Kontakt zum UNHCR und anderen NGOs. Ihm kommt auch eine beratende Funktion zu, und zwar für den Generaldirektor und den Innenminister. Eine wichtige Aufgabe des Ausschusses ist die Überprüfung der Entscheidungen des Refugee Status Determination Officer im Falle eines als offensichtlich unbegründet abgelehnten Asylantrags. Schließlich muß er Studien für eine bessere Behandlung der Flüchtlingsfragen im Lande erstellen. ${ }^{76}$

Die letzte höchste Verwaltungsinstanz für die Behandlung des Asylverfahrens ist der aus drei Personen - mindestens eine davon muß ein qualifizierter Jurist sein - bestehende und vom Innenminister auf fünf Jahre zu ernennende Berufungsbehörde. Die Behörde ist in erster Linie zuständig für die Entscheidung der ihr vorliegenden Rechtsfragen in bezug auf den Refugees Act. Sie entscheidet auch über die Rechtsmittel. Darüber hinaus berät sie den Minister oder den Ständigen Ausschuß in Flüchtlingsfragen. Schließlich liegt die Ernen- 
nungs- und Entlassungskompetenz von Mitgliedern des Ständigen Ausschusses und der Berufungsbehörde ausschließlich in der Hand des Ministers. ${ }^{77}$

\subsubsection{Das Verfahren zur Anerkennung als Flüchtling}

Weder die Genfer Flüchtlingskonvention und deren Protokoll von 1967, noch die OAUKonvention enthalten Aussagen über das Verfahren zur Feststellung der Flüchtlingseigenschaft. Dies wird dem jeweiligen nationalen Recht des Vertragsstaates überlassen. Allerdings muß ein Staat die von EXCOM (UNHCR Executive Committee) in Res. No. 8 (XXVIII) $1977^{78}$ aufgestellten Kriterien - trotz ihrer Unverbindlichkeit - hinsichtlich verfahrensrechtlicher Mindestanforderungen berücksichtigen. Die vom Refugees Act in seinem dritten Kapitel geregelte Struktur des Anerkennungsverfahrens ist dreistufig: Vorverfahren vor der Aufnahmebehörde, Hauptverfahren vor dem Einzelentscheider und Berufungsverfahren. Nach Art. 21 Abs. 1 des Refugees Act hat sich jede Person, die legal oder illegal in Südafrika eingereist ist, in eine der von dem Generaldirektor errichteten und von einem Aufnahmebeamten geführten Empfangsstellen zu begeben und den Asylantrag persönlich zu stellen. Die Aufnahmebeamten - vergleichbar mit den in der Literatur zum deutschen Asylrecht als Anhörer bezeichneten und zum Teil lediglich zur Sachverhaltsermittlung ohne Entscheidungsbefugnis einzusetzenden Beamten ${ }^{79}$ - haben das Gesuch entgegenzunehmen. Da dort lediglich von einer Form die Rede ist - nach $\S 13$ des deutschen AsylVfG liegt z.B. ein Antrag vor, wenn sich dem schriftlichen, mündlichen oder auf andere Weise geäußerten Willen des Ausländers entnehmen läßt, daß er im Bundesgebiet Schutz vor politischer Verfolgung sucht -, ist es anzunehmen, daß ein Asylantrag in Südafrika nur in der vorgeschriebenen Form erfolgen kann.

Der Aufnahmebeamte sorgt dafür, daß der Antrag vollständig ist, indem er die ersten Vorabklärungen vornimmt. Neben der Erhebung der erforderlichen Beweise soll er dabei auch die Gründe, die den Antragsteller davon abhalten, in seine Heimat zurückzukehren, aufnehmen. Aus dem Gesetz geht nicht eindeutig hervor, wo die Anhörung stattfinden soll - es sagt nur "... may conduct such enquiry as he or she deems necessary ..." (auch als "Interview" bezeichnet). Wie der Erläuterung des Innenministeriums zu entnehmen ist, wird das Interview dennoch von dem Aufnahmebeamten durchgeführt. ${ }^{80}$ Anschließend hat er die

Art. 12-14 des Refugees Act.

78

79

Goodwin-Gill, (Anm. 69), S. 472

Vgl. Göbel-Zimmermann, Ralph, Asyl- und Flüchtlingsrecht, Schriftenreihe der NJW, Heft 41/2, 1999, S. 134.

80 S. Home Affairs Portfolio Committee Comments, No. 135-a, in: http://www.polity.org.za/ govdocs/bills/1998.b135-98.pdf. 
Akten dem Einzelentscheider ${ }^{81}$ weiterzuleiten. Somit hat der Aufnahmebeamte im Hinblick auf die Asylgewährung keinerlei materielle Entscheidungsbefugnis. Außerdem stellt er dem Antragsteller - unabhängig von dem Ausgang des Asylgesuchs - eine vorübergehende Aufenthaltsgenehmigung aus. Interessant im Zusammenhang mit der vorübergehenden Aufenthaltsgenehmigung in der eben genannten Erläuterung des Ministeriums ist, daß "unter bestimmten Umständen" die Aufnahme von Arbeit und Studium erlaubt wird.

Nach dem Eingang des Asylgesuchs bei dem zuständigen Beamten entscheidet er über die Anerkennung einer Person als Flüchtling. Der Einzelentscheider hat vier verschiedene Entscheidungsmöglichkeiten: Die Anerkennung eines Flüchtlings als Asylberechtigter (begründeter Antrag), ${ }^{82}$ die Ablehnung eines Antrages als offensichtlich unbegründet, mißbräuchlich oder betrügerisch, ${ }^{83}$ die Ablehnung eines Asylantrages als unbegründet, ${ }^{84}$ und die Weiterleitung des Falls an den Ständigen Ausschuß. ${ }^{85}$

Lehnt der Einzelentscheider einen Antrag ab, so ist zunächst eine behördeninterne Überprüfung möglich. Wird ein Antrag als offensichtlich unbegründet, ${ }^{86}$ mißbräuchlich oder betrügerisch abgelehnt, dann muß der Einzelentscheider dem Antragsteller den Bescheid binnen fünf Arbeitstagen zustellen. In diesem Fall muß der Beamte das Verhandlungsprotokoll mit seinem Ablehnungsgrund an den Ständigen Ausschuß innerhalb von zehn Arbeitstagen nach seiner Entscheidung weiterleiten. ${ }^{87}$

Bei seiner Überprüfung jeder Entscheidung des Einzelentscheiders kann der Ausschuß die angegriffene Entscheidung bestätigen oder aufheben. Bevor er seine Entscheidung trifft, kann er den Vertreter des UNHCR in Südafrika um eine mündliche oder schriftliche Stellungnahme ersuchen oder eine für die Klärung des Sachverhalts als hilfreich in Betracht kommende Person heranziehen. Oder er kann aus eigenem Antrieb eine Befragung oder Untersuchung durchführen. Darüber hinaus kann er den Antragsteller auffordern, vor ihm zu erscheinen und die für den Fall als hilfreich geltenden Informationen zur Verfügung zu stellen. Bei Auslegungsfragen verweist der Ausschuß einen Antrag mit seiner Weisung an

Einzelentscheider nach dem deutschen Asylrecht ( $\$ 5$ Abs. 2 AsylVfG) ist ein der Bediensteter des Bundesamts für die Anerkennung ausländischer Flüchtlinge, der über den einzelnen Asylantrag zu entscheiden hat und nicht weisungsgebunden ist, s. auch Göbel-Zimmermann, (Anm. 79), S. 134.

Art. 24 Abs. 3 (a) des Refugees Act.

Art. 24 Abs. 3 ( b) des Refugees Act.

Art. 24 Abs. 3 (c) des Refugees Act.

Art. 24 Abs. 3 (d) des Refugees Act.

Das Gesetz definiert einen "offensichtlich unbegründeten" Antrag als "... an application for asylum made on grounds other than those on which an application may be made under this Act", vgl. Art. 1 Abs. (xii) des Refugees Act.

Art. 24 Abs. 4 (b) des Refugees Act. 
den Einzelentscheider zurück. Der Einzelentscheider hat den Antrag auf der Basis dieser Weisung zu entscheiden. ${ }^{88}$ Damit kommt dem Ausschuß neben der Revision auch eine Kontrollfunktion zu.

Der Antragsteller selbst kann auch gegen die Ablehnung seines Anerkennungsantrages durch den Einzelentscheider innerhalb von zehn Tagen nach Zustellung des Bescheids ein schriftliches Rechtsmittel bei der Berufungsbehörde einlegen. Nach der Prüfung des Rechtsmittels kann die Berufungsbehörde die Entscheidung des Einzelentscheiders bestätigen oder aufheben. Sie kann die Vertretung des UNHCR im Lande ersuchen und sie um mündliche oder schriftliche Stellungnahme bitten. Des weiteren kann sie die Angelegenheit an den Einzelentscheider zwecks weiterer Sachaufklärung zurückverweisen oder eine solche selbst durchführen lassen. Dabei muß die Behörde einen Vertreter des Asylsuchenden in Rechtssachen zulassen. ${ }^{89}$ Die Entscheidung der Berufungsbehörde gilt als die endgültige und letzte Stufe der behördeninternen Überprüfung im System des Refugees Act. $\mathrm{Ob}$ die von dem Einzelentscheider erlassenen Bescheide und die darauffolgenden administrativen Entscheidungen gerichtlich angreifbar und überprüfbar sein können, ist dem Text des Gesetzes nicht zu entnehmen. Wenn man dem Innenministerium Glauben schenken darf, stünde einem Asylantrag nach der Ausschöpfung des administrativen Verfahrens der Gang zu den Verwaltungsgerichten weiterhin offen. ${ }^{90}$ Das Verwaltungsverfahren soll nach Angaben des Innenministeriums sechs Monate dauern, während für die Revision lediglich drei Monate ausreichen sollen.

\subsection{Die Rechtsstellung der anerkannten Flüchtlinge}

\subsubsection{Rechte der anerkannten Flüchtlinge}

In seinem dritten Kapitel listet das Gesetz Rechte und Pflichten der anerkannten Flüchtlinge in Südafrika auf. Da der Refugees Act sich in seiner Präambel zu den Standards und Prinzipien der Genfer Flüchtlingskonvention, dem dazugehörigen Protokoll von 1967, der OAU-Konvention und allen anderen Menschenrechtsdokumenten bekennt, ${ }^{91}$ kommt ein anerkannter Flüchtlinge in den vollen Genuß der im Kapitel zwei der Verfassung (Bill of Rights) für Bürger Südafrikas verankerten Rechte. Diese Rechte entsprechen den Bestim-

Art. 25 Abs. 1-5 des Refugees Act.

S. Home Affairs Portfolio Committee Comments, (Anm.80).

Art. 6 Abs. 1 Refugees Act sieht vor, daß für dessen Interpretation außer den oben genannten internationalen Vertragswerken, die Allgemeinen Erklärung der Menschenrechte aus dem Jahre 1948 und alle relevanten Konventionen oder internationalen Vereinbarungen, deren Vertragspartei Südafrika ist oder wird, herangezogen werden müssen. 
mungen der Genfer Flüchtlingskonvention. Demnach erhalten Asylsuchende in Südaf rika nach ihrer Anerkennung als Flüchtlinge eine ständige Aufenthaltserlaubnis, eine Identitätskarte und einen Reiseausweis. Im übrigen sollen sie im Arbeits-, Gesundheits- und Bildungswesen südafrikanischen Staatsangehörigen gleichgestellt sein. ${ }^{92}$

Nicht ganz mit der Bestimmung der Genfer Flüchtlingskonvention in Einklang zu bringen ist allerdings Art. 7 (C) des Refugees Act. Entgegen Art. 34 der Genfer Flüchtlingskonvention, der die Vertragschließenden Staaten in die Pflicht nimmt, die Eingliederung und Einbürgerung der Flüchtlinge soweit wie möglich zu erleichtern, sieht der Refugees Act lediglich vor, daß ein anerkannter Flüchtling nach fünfjährigem ununterbrochenen Aufenthalt in Südafrika einen Immigrationsantrag stellen darf, wenn der Ständige Ausschuß ihm bescheinigt, daß seine Eigenschaft als Flüchtling unbestimmt fortdauern wird.

Die Rechte der Flüchtlinge unterliegen jedoch der öffentlichen Sicherheit oder Ordnung ("national security or public order") Südafrikas. Im Falle eines Verstoßes gegen die öffentliche Sicherheit oder Ordnung des Landes ermächtigt der Refugees Act den Innenminister, einen Flüchtling unter gebührender Berücksichtigung der Bestimmungen des Art. 33 der Verfassung Südafrikas ${ }^{93}$ und des internationalen Flüchtlingsrechts auszuweisen. Außerdem darf der Innenminister die Verwahrung eines auszuweisenden Flüchtlings in der Abschiebehaft bis zu dreißig Tagen anordnen, um ihm eine angemessene Frist ("reasonable time") einzuräumen, ein anderes Land seiner Wahl aufsuchen zu können. ${ }^{94}$ Es wird nirgends ein Hinweis darauf gegeben, was unter "öffentlicher Sicherheit oder Ordnung" zu verstehen ist. Hinzu kommt, daß nicht klar wird, welche rechtlichen Möglichkeiten ein Flüchtling hat, sich gegen eine solche Maßnahme gerichtlich zu Wehr zu setzen. Das Gesetz sieht lediglich vor, daß beim Überschreiten der dreißigtägigen Frist die Haft vom High Court Richter überprüft werden muß. Es ist zweifelhaft, ob diese Regelung mit Art. 35 der Verfassung vereinbar ist. Dort wird unmißverständlich geregelt, daß eine inhaftierte Person sobald wie möglich nach ihrer Verhaftung, jedenfalls innerhalb von achtundvierzig Stunden, einem Gericht vorzuführen ist. Das Gesetz schweigt darüber, was aus dem Flüchtling werden wird, wenn er innerhalb der "reasonable time" kein Aufnahmeland finden kann. Diese nicht eindeutige Einschränkung der Rechte eines anerkannten Flüchtlings könnte der Exekutive Tür und Tor öffnen, sich mit der Ermächtigung des Refugees Act im Rücken unliebsamer Flüchtlinge - aus welchen Gründen auch immer - außergerichtlich entledigen zu können.

92

93

94
Art. 33 der Verfassung regelt ein rechtmäßiges und gerechtes Verwaltungshandeln (just administrative action).

Art. 28 Abs.1-5 des Refugees Act. 
Eine bemerkenswerte Bestimmung ist die Regelung für Kinder ohne Begleitung und für psychisch kranke Menschen. In Bezugnahme auf die Kinderschutz-Konvention von $1989^{95}$ regelt der Refugees Act, daß der Fall eines die Flüchtlingsdefinition des Art. 3 des Refugees Act zu erfüllen scheinenden schutzsuchenden Kindes unverzüglich vor ein Jugendgericht gebracht werden muß. Das Gericht kann dann anordnen, dem Kind bei der Antragstellung Hilfe zukommen zu lassen. Ähnlich ordnet Art. 32 Abs. 3 des Refugees Act an, daß einem geistig kranken Flüchtling, der die Voraussetzungen der Flüchtlingseigenschaft zu erfüllen scheint, bei der Asylantragstellung geholfen werden muß. ${ }^{96}$

Familienangehörigen eines Asylberechtigten gewährt der Refugees Act ein Aufenthaltsrecht in Südafrika. Im Falle einer Heirat oder Erlangung der Volljährigkeit geht das Aufenthaltsrecht nicht verloren. ${ }^{97}$ Ähnliches gilt auch bei Scheidung und in Todesfällen. Nach dem Tod eines Asylberechtigten wird Hinterbliebenen, die unmittelbar vor dem Eintritt des Todesfalls mit dem nun Verstorbenen gelebt haben, weiterhin das Aufenthaltsrecht in Südafrika gewährt. Dies gilt auch in Scheidungsfällen. ${ }^{98}$ Einem eigenen Anerkennungsverfahren des Angehörigen steht auch im übrigen nichts im Wege. ${ }^{99}$

\subsubsection{Pflichten eines Asylberechtigten}

Als die Pflichten eines Asylberechtigten schreibt das Gesetz vor, daß die Person sich den Gesetzen Südafrikas unterwerfen muß. ${ }^{100}$

\subsubsection{Regeln zur Beendigung des Flüchtlings-Status}

Das Asylgesetz nennt fünf Gründe für die Beendigung des Asyls, die sowohl mit den Bestimmungen des Art. I Abs. 4 der OAU-Konvention wie auch des Art. 1 Abschnitt C Genfer Flüchtlingskonvention weitgehend übereinstimmen. Demnach endet das Asyl durch einen freiwilligen Wiedererwerb des Schutzes des Staates, dessen Staatsangehörigkeit der Flüchtling besitzt. Nach Art. 5 Abs. 1 b findet das Asylgesetz keine Anwendung mehr auf einen Flüchtling, wenn er nach dem Verlust seiner Staatsangehörigkeit diese freiwillig wiedererlangt hat. Des weiteren stellt der Genuß einer neu erworbenen Staatsangehörigkeit und der Schutz des Landes, dessen Staatsangehörigkeit ein Flüchtling erworben hat, einen

Südafrika ist mit seiner Ratifikation 1995 dem Übereinkommen über die Rechte des Kindes vom 20.11.89 beigetreten, s. Anm. 15 .

96

97

98

99

100
Art. 32 Abs. 2 des Refugees Act.

Art. 33 Abs. 2 des Refugees Act

Art. 33 Abs. 3 des Refugees Act.

Art. 33 Abs. 4 des Refugees Act.

Art. 34 des Refugees Act. 
Beendigungsgrund der Flüchtlingseigenschaft dar. Ferner gilt die freiwillige Rückkehr ins Heimatland, das der Flüchtling aus Furcht vor Verfolgung verlassen hat, oder die Annahme der Staatsangehörigkeit Südafrikas als weiterer Beendigungsgrund. Schließlich führt der Wegfall der Umstände, aufgrund derer eine Person als Flüchtling anerkannt worden ist wenn sie nicht mehr ablehnen kann, den Schutz des Landes in Anspruch zu nehmen, dessen Staatsangehörigkeit sie besitzt - zur Beendigung des Schutzes als Flüchtling.

\subsection{Der Grundsatz des Non-refoulement}

Der Refugees Act bekennt sich zu dem vom UNHCR als "Kernstück des internationalen Schutzsystems" 101 charakterisierten und heute im Völkerrecht weitgehend verankerten Prinzip des Non-refoulement ${ }^{102}$ (Verbot der Zurückweisung, Abschiebung oder Ausweisung in den Verfolgerstaat). Dabei richtet er sich in seinem Art. 2 nach den Bestimmungen des Art. 33 der Genfer Flüchtlingskonvention. So verbietet das Gesetz, einer Person den Zugang zu Südafrika zu verwehren, sie des Landes zu verweisen, auszuliefern oder sie in irgendein Land zurückzuweisen, wenn sie aufgrund dessen gezwungen wäre, in ein Land zurückzukehren oder in einem Staat zu bleiben, wo sie wegen ihrer Rasse, Religion, Nationalität, Zugehörigkeit zu einer bestimmten sozialen Gruppe oder ihrer politischen Überzeugung Verfolgungen ausgesetzt sein könnte oder wo ihr Leben, ihre körperliche Unversehrtheit oder Freiheit infolge einer von außen kommenden Aggression, ausländischer Besetzung oder Fremdherrschaft, oder schwerwiegender Störungen der öffentlichen Sicherheit und Ordnung gefährdet wäre.

\subsection{Sonstige Bestimmungen}

\subsubsection{Gruppenanerkennungsverfahren}

Neben den oben angeführten Anerkennungsverfahren für individuelle Asylanträge regelt der Refugees Act in seinem letzten Kapitel auch die Aufnahme und Unterbringung von Asylsuchenden im Falle von großen Flüchtlingsgruppen oder im Falle eines Massenzustroms, ein sog. Gruppenanerkennungsverfahren. In Fällen, wo eine Gruppe oder eine Kategorie von Personen in Südafrika eintrifft und sie vom Innenminister als Flüchtlinge im

101 UNHCR (Hrsg.), Zur Lage der Flüchtlinge in der Welt, 1994, S. 5.

102

$\mathrm{Zu}$ dem Meinungsstreit hinsichtlich der Einstufung des Non-refoulement-Prinzips als Norm des Völkergewohnheitsrechts, s. Goodwin-Gill, (Anm. 69), S. 117 ff; Gornig, Gilbert, Das nonrefoulement"-Prinzip, ein Menschenrecht "in statu nascendi", in: EuGRZ 18/1986, S. 521-529 (525); Hailbroner, Kay, Refoulement and "Humanitarian" Refugees: Customary International Law or Wishful Legal Thinking?, in: David Martin (Hrsg.), The New Asylum Seekers: Refugee Law in the 1980s, 1988, S. $128 \mathrm{f}$. 
Sinne der Definition des Art. 3 des Refugees Act betrachtet werden können, wird der Minister dazu ermächtigt, diese Personen zu Flüchtlingen zu erklären und dies im Amtsblatt zu veröffentlichen. ${ }^{103}$ Die Erklärung des Ministers kann entweder vorbehaltlos oder aber widerrufbar sein. Da in solchen Fällen auf ein individuelles Asylverfahren verzichtet und die kollektive Flüchtlingseigenschaft in Betracht gezogen wird, hat eine solche Vorgehensweise einen provisorischen humanitären Charakter. Aus praktischen Erfahrungen in Afrika, wo es häufig zum Zustrom von sehr großen Flüchtlingsgruppen kommt, ist die Aufnahme dieser Regelung in das nationale Gesetz von praktischer Bedeutung.

\subsubsection{Widerruf einer Anerkennung}

Art. 36 des Refugees Act regelt den Widerruf einer Anerkennung, falls sich herausstellt, da $ß$ eine Person aufgrund eines unrichtigen und auf falschen Informationen beruhenden Antrags oder durch Täuschung, Fälschung oder falsche oder irreführende Darstellung ihrer Lage anerkannt wurde. In einem solchen Fall widerruft der Ständige Ausschuß den Schutz des anerkannten Flüchtlings. Danach wird diese nach dem Aliens Control Act von 1991 als "prohibited person" behandelt.

Art. 37 des Refugees Act widmet sich den zu verhängenden Geld- und Freiheitsstrafen im Falle einer illegalen Einreise oder Aufenthalts in Südafrika. Danach wird der Verstoß gegen das Gesetz entweder bei illegaler Einreise oder bei Hilfeleistung für solche Handlungen mit Freiheitsentzug bis fünf Jahren oder Geldstrafe oder beidem bestraft.

\subsubsection{Ausführungsverordnungen}

Zusätzlich zu den bereits genannten umfassenden Befugnissen des Innenministers wird er durch Art. 38 des Refugees Act ermächtigt, Ausführungsverordnungen zu den Regelungen des Gesetzes zu erlassen. Die Verordnungen sollen einen Flüchtlingszustrom großen Ausmaßes, Art und Weise sowie die Frist, innerhalb derer ein Fall an den Ständigen Ausschuß verwiesen werden muß, regeln.

In Art. 39 des Refugees Act wird dem Generaldirektor die Pflicht auferlegt, in Beratung mit dem Ständigen Ausschuß Maßnahmen zu ergreifen, um Personen, die mit der Verwirklichung der Bestimmungen des Refugees Act betraut sind, angemessen und umfassend auszubilden.

Schließlich enthalten die letzten beiden Artikel des Gesetzes Übergangsbestimmungen und Wirksamkeitsregelungen. 


\section{Ausblick}

Mit dem Erlaß des Refugees Act vom 2. Dezember 1998 hat Südafrika eine umfassende rechtliche Grundlage für die Gewährung von Asyl für Flüchtlinge geschaffen. Festzustellen bleibt jedoch, daß mit diesem Asylgesetz kein subjektiver Anspruch auf Asyl eingeführt worden ist. Trotz einiger abweichender Bestimmungen in dem Refugees Act, die mit dem internationalen Flüchtlingsrecht nicht in Einklang zu bringen sind, stimmt das Gesetz grundsätzlich mit den wichtigsten Voraussetzungen des Flüchtlingsschutzes überein. Daher stellt das Gesetz eine Bereicherung sowohl des afrikanischen als auch des internationalen Flüchtlingsrechts dar. Insbesondere die Aufnahme internationaler menschenrechtlicher Verträge in das Gesetz, wie die Allgemeine Erklärung der Menschenrechte aus dem Jahre 1948 - wenngleich ihr keine Bindungskraft zukommt - ist ein begrüßenswerter und beispielhafter Schritt. Damit bekennt sich Südafrika zu dem Konzept, daß Flüchtlingsschutz untrennbar mit der Idee der Menschenrechte verbunden ist.

Wie die Erfahrung lehrt, hängt ein funktionierendes Flüchtlingsschutzsystem weitgehend von der Schaffung einer kompetenten und vom Einfluß der Regierung unabhängigen Behörde ab. Diese Unabhängigkeit des Asylverfahrens ist auch eine der wichtigsten Forderungen des Draft Green Paper on International Migration gewesen: "Refugee status determination should be the domain of an expert authority with reasonable assurance of independence from both the executive and political branches of government". ${ }^{104} \mathrm{Da}$ das neue Flüchtlingsgesetz auf der alten Struktur des Ausländergesetzes (Aliens Control Act) aufgebaut wurde und neben der südafrikanischen Polizei, die für Willkür und Mißhandlungen von Ausländern bekannt ist, auch die Mitarbeiter des Department of Home Affairs, die mit Flüchtlingsanträgen zu tun hatten, als korrupt ${ }^{105}$ galten, hängt die Effektivität des Refugees Act weitgehend von den im Gesetz ausdrücklich vorgesehenen Trainings des Personals ab, das speziell für Aufgaben im Umgang mit Flüchtlingen ausgebildet werden soll.

Zusammenfassend läßt sich feststellen, daß die normativen Bestimmungen des neuen und ersten Flüchtlingsgesetzes Südafrikas als ebenso fortschrittlich und liberal zu bezeichnen sind, wie die von den Kommentatoren als sehr modern, ${ }^{106}$ fortschrittlich und schutzfreund-

104

105 Die Untersuchung von Human Rights Watch schildert die Korruption der Beamten des Department of Home Affairs folgendermaßen: "We discovered extensive corruption in the refugee determination process, with Home Affairs officials demanding bribes for the scheduling of inter106 views and for the granting of permits, vgl. Human Rights Watch, (Anm. 41).

Vgl. Hofmann, Rainer: Das neue Flüchtlingsgesetz von Simbabwe, in: Jahrbuch für Afrikanisches Recht, 5/84, S. 67-77 (67). 
lich $^{107}$ gepriesenen Flüchtlingsgesetze Simbabwes und Lesothos. Von daher muß dem südafrikanischen Flüchtlingsgesetz auch zukünftig besondere Aufmerksamkeit geschenkt werden.

107 UNHCR (Hrsg.), Flüchtlinge, Nr. 2, Juni 1994, S. 9 f. 
be better suited to prevent these types of destructive behaviour than traditional commandand-control regulation.

The underlying thought is that private property rights provide an incentive for efficient exploitation and allocation of scarce resources because the right of the owner to capture the benefits accruing from the resource provides an incentive to invest in the resource.

The paper proposes a market solution on the basis of Biodiversity Property Rights (BDPR) with at least two parties, the local supplier (BDS) and the demander (BDD). Stress is placed on the need for the contractual framework to be adjusted to differing circumstances and required incentives. The paper further focuses on the legal characteristics of the BDPR and the obligations of the BDD-party and BDS party.

\section{South Africa's Refugees Act 1998}

\section{By Tamru Melese}

Until only a few years ago, Southern Africa has been one of the two regions, besides the conflict-ridden Horn of Africa, that produced the majority of refugees in Africa. Most of the refugees in Southern Africa were the product of the white-minority apartheid regime in South Africa.

Its aggressive destabilization of neighbouring countries in the region, particularly Angola and Mozambique, forced several hundred thousand people to flee their homes. With the dramatic political changes that have taken place in the region over the last few years, Southern Africa ceased to be a major refugee-producing region. Since the first all-races democratic elections in 1994, South Africa is being flooded with requests for asylum.

The post-apartheid South Africa made substantial progress towards establishing a legal system based on respect for the human rights. Based on the new democratic Constitution, South Africa ratified several international treaties on human rights. In order to bring the country in line with its international, regional and constitutional obligations, to provide for the reception into South Africa of asylum seekers, to regulate applications for recognition of refugee status, and to provide for the rights and obligations flowing from such status, the post-apartheid South African government has committed itself to the development of a refugees act. Accordingly, the Refugees Act 130 of 1998 has been enacted on December 2, 1998.

The Act sets clear guidelines, in acknowledging international human rights instruments and in keeping with principles of the 1951 Geneva Convention and the 1969 OAU Convention. As a result, the refugee definition in the Refugees Act is generous, and includes the key provisions of the above mentioned conventions. Therefore, the definition of the Act acknowledges the individualized refugee status determination procedures as well as a 
collectivized protection approach. The author comes to the conclusion that, generally speaking, the Refugees Act is largely consistent with international conventions and the majority of its provisions satisfy the formal international legal requirements.

\section{Tax Justice in German and Turkish Constitutional Law}

\section{By Joachim Lang}

The article contains a comparative study of those provisions of the German and Turkish constitutions that deal with tax justice. Not surprisingly, both constitutions share the same basic ideas, because the constitution of the Republic of Turkey of 1982 as well as the German constitution have a common ancestor: the French Declaration of Human and Civil Rights of 1789. In particular, both constitutions take up the internationally acknowledged concept of tax justice, the so-called ability-to-pay-principle (i.e. the fair distribution of the tax burden in accordance with each taxpayer's economic ability). On a more specific level, both constitutions utilize similar concepts to define the cornerstones of their respective tax systems: progressive taxation of income, protection against excessive taxation, taxation as a means to finance welfare programs (redistribution of wealth), and preferred tax treatment of married taxpayers and families, which enjoy special constitutional protection. There is, however, one significant difference: While the Turkish constitution sets forth a strict separation of church and state, Germany has always rejected pure laicism. As a result, certain denominations (e.g., the Catholic and the Protestant Church) and some religious communities are granted a special status by the German constitution. 